\title{
A Modified Acetylcholine Receptor $\delta$-Subunit Enables a Null Mutant to Survive Beyond Sexual Maturation
}

\author{
Kimberly E. Epley, ${ }^{1 *}$ Jason M. Urban, ${ }^{2 *}$ Takanori Ikenaga, ${ }^{2}$ and Fumihito Ono ${ }^{2}$ \\ ${ }^{1}$ The Whitney Laboratory for Marine Bioscience, University of Florida, St. Augustine, Florida 32080, and ${ }^{2}$ Section on Model Synaptic Systems, Laboratory of \\ Molecular Physiology, National Institute on Alcohol Abuse and Alcoholism-National Institutes of Health, Bethesda, Maryland 20892
}

The contraction of skeletal muscle is dependent on synaptic transmission through acetylcholine receptors (AChRs) at the neuromuscular junction (NMJ). The lack of an AChR subunit causes a fetal akinesia in humans, leading to death in the first trimester and characteristic features of Fetal Akinesia Deformation Sequences (FADS). A corresponding null mutation of the $\delta$-subunit in zebrafish (sofa potato; sop) leads to the death of embryos around $5 \mathrm{~d}$ postfertilization (dpf). In $s o p^{-1-}$ mutants, we expressed modified $\delta$-subunits, with one ( $\delta 1 \mathrm{YFP}$ ) or two yellow fluorescent protein ( $\delta 2$ YFP) molecules fused at the intracellular loop, under the control of an $\alpha$-actin promoter. AChRs containing these fusion proteins are fluorescent, assemble on the plasma membrane, make clusters under motor neuron endings, and generate synaptic current. We screened for germ-line transmission of the transgene and established a line of sop ${ }^{-1-}$ fish stably expressing the $\delta 2 \mathrm{YFP}$. These $\delta 2 \mathrm{YFP} / \mathrm{sop}^{-1-}$ embryos can mount escape behavior close to that of their wild-type siblings. Synaptic currents in these embryos had a smaller amplitude, slower rise time, and slower decay when compared with wild-type fish. Remarkably, these embryos grow to adulthood and display complex behaviors such as feeding and breeding. To the best of our knowledge, this is the first case of a mutant animal corresponding to first trimester lethality in human that has been rescued by a transgene and survived to adulthood. In the rescued fish, a foreign promoter drove the transgene expression and the NMJ had altered synaptic strength. The survival of the transgenic animal delineates requirements for gene therapies of NMJ.

Key words: zebrafish; neuromuscular junction; acetylcholine receptor; synapse; fetal akinesia deformation sequence; fluorescent protein

\section{Introduction}

The synapse between the motor nerve and skeletal muscle, commonly referred to as the neuromuscular junction (NMJ), is cholinergic in vertebrates and is a model system for the investigation of synapses (Sanes and Lichtman, 2001; Ono, 2008). On the postsynaptic face of the NMJ, the AChRs span the membrane, with each AChR forming a pentameric structure. The five subunits comprising AChRs are $2 \alpha s, \beta, \delta$ and $\gamma / \varepsilon$. $\gamma$ and $\varepsilon$ are developmentally regulated and can substitute for each other. The embryonic-type $\gamma$ is replaced by the adult-type $\varepsilon$ as the synapse matures (Mishina et al., 1986).

A group of genetic disorders have mutations in genes coding for components of the NMJ. Congenital myasthenic disorders (CMDs) result from mutations in genes coding for expression of

Received June 19, 2008; revised 0ct. 14, 2008; accepted 0ct. 16, 2008.

This work was supported by National Institute of Neurological Disorders and Stroke Grant 1R01NS050388-01A1 to F.O. and K.E.E., Muscular Dystrophy Association Grant MDA3818 to F.0., and the intramural research program of the National Institute on Alcohol Abuse and Alcoholism-National Institutes of Health. We thank Elizabeth Jimenez, Nichole Gebhart, Karen Overstreet, and Anna Mistretta-Bradley for their help in the maintenance of the zebrafish facility. We thank Alison Delargy, Jessica Roberts-Misterly, Rebecca Price, and Jim Netherton for their help in the manuscript editing, clone constructs, setting-up the electrophysiology and the digital imaging of adult fish, respectively. We thank Dr. Stephen Ikeda for critical reading of this manuscript. SV2 antibody, developed by Dr. Kathleen Buckley, was obtained from the Developmental Studies Hybridoma Bank developed under the auspices of the National Institute of Child Health and Human Development and maintained by the University of lowa.

*K.E.E. and J.M.U. contributed equally to this work.

Correspondence should be addressed to Fumihito Ono, MSC9411, National Institute on Alcohol Abuse and Alcoholism-National Institutes of Health-Bethesda, MD 20892. E-mail: onof@mail.nih.gov.

DOI:10.1523/JNEUROSCI.2814-08.2008

Copyright $\odot 2008$ Society for Neuroscience $\quad 0270-6474 / 08 / 2813223-09 \$ 15.00 / 0$ proteins such as AChR, rapsyn, MuSK, and cholinesterase (Engel and Sine, 2005). CMD patients display weak muscle strength resulting from compromised synaptic currents. Functional nulls of AChRs were predicted to be lethal and, indeed, fetuses homozygous for these mutations die in the first trimester (Michalk et al., 2008). Affected fetuses display characteristic anatomical features that are collectively called Fetal Akinesia Deformation Sequences (FADS).

Zebrafish (Danio rerio) emerged as a model for NMJ studies after a large-scale mutagenesis screening identified mutants displaying defects in neuromuscular transmission (Granato et al., 1996). In this screening, the zebrafish genome was randomly mutated with $\mathrm{N}$-ethyl- $\mathrm{N}$-nitrosourea (ENU), and homozygous embryos that displayed specific behavioral phenotypes were isolated. Homozygous sofa potato $\left(\right.$ sop $\left.^{-1-}\right)$ embryos do not exhibit any movement. The causal mutation was identified in the AChR $\delta$-subunit, as a leucine to proline substitution near the $\mathrm{N}$ terminus (Ono et al., 2004). Mutant $\delta$-subunits do not form pentamers and, thus, receptors do not reach the plasma membrane. Sop ${ }^{-\prime-}$ embryos show no AChRs on the muscle cell surface and synaptic recordings from muscle cells reveal no currents (Ono et al., 2001). These embryos eventually die $\sim 5 \mathrm{~d}$ postfertilization (dpf), without hatching out of the chorion. Thus, in terms of genetics and physiology, Danio rerio sop ${ }^{-1-}$ mutants are counterparts to the $\delta$-subunit mutation in humans.

Here, we report a transgenic zebrafish with a $s o p^{-1-}$ background in which all functional AChRs have insertions of one or 
two YFP molecules attached to the long intracellular loop of the $\delta$-subunit. The embryos displayed strong fluorescence at NMJs. The synaptic development and physiology of embryos with these modified $\delta$-subunits were studied. These embryos survived well into adulthood and displayed normal behaviors.

\section{Materials and Methods}

Fish strains. Sofa potato mutant, sop $\mathrm{tj}^{19 \mathrm{~d}}$, was originally obtained from the Max Plank Institute, and maintained in the animal facilities at the University of Florida and National Institute on Alcohol Abuse and Alcoholism-National Institutes of Health (NIH). Adult fish were maintained in stand-alone, self-circulating AHAB systems (Aquatic Ecosystems) following Institutional Animal Care and Use Committee guidelines at the University of Florida and NIH. Embryos obtained from crosses were reared at $28^{\circ} \mathrm{C}$.

DNA construct and the injection of DNA construct into zebrafish embryos. The construct for the expression of zebrafish AChR $\delta$-subunit contained in sequence: Megalinker sequence (Thermes et al., 2002), $\alpha$-actin promoter (Higashijima et al., 1997), $\delta$-subunit (Ono et al., 2004), and polyadenylation signal sequence. The $\delta$-subunit sequence contained an inserted yellow fluorescent protein (YFP) sequence in frame, such that one or two YFPs were added in the intracellular loop of the $\delta$-subunit (see Fig. $1 a, b)$. The YFP fragment, with additional AgeI sites at both ends, was ligated into the AgeI site of the $\delta$-subunit gene. The generated clone was verified by sequencing. Injection of DNA construct into fertilized zebrafish eggs was performed as previously described, with slight modifications (Ono et al., 2004). The pipette solution contained circular plasmid $10 \mu \mathrm{g} / \mathrm{ml}, 0.5 \times$ Reaction Buffer, and I-SceI $1 \mathrm{U} / \mu \mathrm{l}$ (Roche). Injection was performed on an agarose gel with a glass microelectrode connected to a syringe. Fertilized eggs were injected before the first cell division.

For establishment of stable lines expressing $\delta 2$ YFP, DNA-injected embryos were raised to adults. Reproductively mature adults were set-up for in- or out-crossing. Embryos were screened for fluorescence in muscle cells using a Zeiss SV11 fluorescent microscope (Carl Zeiss Microimaging). Four adults carrying the transgene in the germline were established as independent lines and used to generate offspring in $s o p^{+/-}$background. Two adults, each with one allele of the sop mutation, were mated to obtain $\delta 2 \mathrm{YFP} / \mathrm{sop}^{-1-}$ embryos for experiments.

Genotyping of sop ${ }^{-/-}$fish. Two methods were combined to genotype and confirm the $s o p^{-1-}$ mutation. In the first method, we used the restriction enzyme BccI (New England Biolabs). Primers used for PCR were: AGGAGTATGACATTAAGG (Forward) and TGGAACCACTTGAGGTTG (Reverse). Both primers correspond to sequences in introns of the $\delta$-subunit gene (see Fig. 1c). After genome extraction, PCR was performed using genomic DNA as a template. Amplified DNA was subsequently digested with BccI and run on a $2 \%$ agarose gel (see Fig. $1 d$ ). The uncut, wild-type amplicon is $397 \mathrm{bps}$, and BccI digestion of the sop sequence generates two shorter fragments, 181 bps and 214 bps. In the second method, primers TCTTGAAATAGGTCTGACTTGCAG (Forward) and GCAATATTAAGCACTCACCAAGG (Reverse) were used to amplify a $279 \mathrm{bp}$ sequence from the extracted genome. After purification, the PCR product was submitted for direct sequencing (Macrogen) using the reverse primer.

Video imaging. High-speed video imaging of $3 \mathrm{dpf}$ embryos was performed using the Photron 1024PCI camera (TechImaging) mounted on a Zeiss stereomicroscope Stemi 2000-C. Images were taken at the rate of 500 or 1000 frames per second (fps). Sequential images were stored and later processed in Photoshop (Adobe).

For the analysis of movement (see Fig. $4 d$ ), we followed methods of Liu and Fetcho (1999) with some modifications. Sequential images were imported to NIH ImageJ. A rostral midline, of a fixed length, was drawn from the tip of the head toward the center of the yolk sac. Coordinates for the head tip in the $1024 \times 1024$ space and the angle of the rostral midline were measured at individual time points. Data were exported to IGORPro (WaveMetrics), and the movement of the rostral midline was plotted. All embryos were siblings from a single adult pair and the genotype was determined after video recording. Student's $t$ test ( $p \leq 0.05$ ) was used to compare parameters.

Still images of adult fish were taken with a conventional digital camera.

Fluorescent imaging. Low magnification fluorescent imaging of zebrafish embryos was performed on a Zeiss SV11 microscope (Zeiss) with the filter cube optimized to detect YFP fluorescence. YFP was excited using a Mercury lamp. Digital images were acquired with a color CCD camera (DP70; Olympus) and processed with Photoshop.

Confocal images were taken on a SP2 confocal microscope (Leica Microsystems) or LSM510 Meta (Zeiss) using a $25 \times$ (numerical aperture 0.8 ) water-immersion objective. YFP was excited with the $514 \mathrm{~nm}$ laser line. Triple staining, fixation and immunohistochemistry of YFPpositive embryos with $\alpha$-bungarotoxin $(\alpha$-Btx) and anti-SV2 antibody were performed as previously described (Ono et al., 2004). YFP, Alexa555 ( $\alpha$-Btx), and Cy5 (SV2) were excited with 488, 561 and $633 \mathrm{~nm}$ laser lines, respectively. Emission spectra were adjusted and sequential scanning mode was used to minimize the crosstalk of signals. Obtained images were analyzed with Photoshop.

Electrophysiology. Electrophysiology was performed on an Olympus BX51WI microscope (Olympus) following protocols as previously described (Ono et al., 2002) with slight modifications. The bath solution contained $\mathrm{NaCl} 110 \mathrm{~mm}$, HEPES $5 \mathrm{~mm}, \mathrm{CaCl}_{2} 2 \mathrm{~mm}$, glucose $3 \mathrm{~mm}, \mathrm{KCl} 2$ $\mathrm{mm}, \mathrm{MgCl}_{2} 0.5 \mathrm{~mm}$, and $18 \beta$-glycyrrhetinic acid $100 \mu \mathrm{M}, \mathrm{pH}$ 7.4. $18 \beta$ glycyrrhetinic acid was added to block the electrical coupling of muscle cells through gap junctions (Luna et al., 2004). The pipette solution contained $\mathrm{KCl} 120 \mathrm{~mm}$, HEPES $5 \mathrm{~mm}$, and BAPTA $5 \mathrm{~mm}$, pH 7.1. Muscle cells were voltage clamped at $-90 \mathrm{mV}$ for $\mathrm{mEPC}$ recordings. Signals were collected with an AD/DA converter ITC18 (Instrutech), Axopatch 1C amplifier (Molecular Devices) and Pulse program (Heka). Signals were sampled at $100 \mathrm{kHz}$ and filtered at $5 \mathrm{kHz}$. Muscle cells were identified as fast-twitching fibers or slow-twitching fibers based on their location and orientation. mEPCs were analyzed using MiniAnalysis (Synaptosoft). The Mann-Whitney $U$ test $(p \leq 0.05)$ was used to compare mEPC parameters (i.e., rise time, decay time constant, and current amplitude) between $\delta 2$ YFP and wild type. Evoked synaptic currents were recorded following methods described by Ono et al. (2002).

Biochemistry. Embryos (6dpf) were mechanically deyolked and immediately transferred to cold RIPA buffer (25 mM Tris-HCl pH 7.6, $150 \mathrm{~mm}$ $\mathrm{NaCl}, 1 \% \mathrm{NP}-40,1 \%$ Sodium deoxycholate and $0.1 \%$ SDS) containing protease and phosphatase inhibitors (Pierce). Samples were homogenized on ice and subsequently spun down at $5000 \times g$, the supernatant was removed and mixed with NuPAGE LDS Sample Buffer containing $10 \%$ Sample Reducing Agent (Invitrogen). After incubation at $70^{\circ} \mathrm{C}$ for $10 \mathrm{~min}$, protein samples were spun down again and supernatant was separated on a NuPAGE Novex $4-12 \%$ Bis-Tris gel (Invitrogen) with MOPS running buffer then transferred to polyvinylidene difluoride (PVDF) membrane (Invitrogen). All incubations were performed for $1 \mathrm{~h}$ at room temperature with constant shaking. Blots were blocked with $5 \%$ bovine serum albumin (Sigma) in Tris-buffered saline (TBS; $10 \mathrm{~mm}$ Tris$\mathrm{HCl}, 150 \mathrm{~mm} \mathrm{NaCl}, \mathrm{pH} \mathrm{7.5)}$ and $0.1 \%$ Tween 20. Primary antibody incubation was performed with anti-GFP ( $\mathrm{N}$ terminal, 1:1500; Sigma), blots were washed and then incubated with HRP conjugated Goat antiRabbit IgG (1:3000, Sigma). Signal was detected using SuperSignal West Pico chemiluminescent substrate kit (Pierce) on Amersham Hyperfilm ECL (GE Healthcare). Chemiluminescent images were captured using a Kodak Image Station 4000R (Kodak).

Histochemistry of adult zebrafish. Adult fish were anesthetized in $0.1 \mathrm{~g} / \mathrm{l}$ MS-222 (tricaine methanesulfonate; Sigma) before decapitation. The trunk was dissected into small pieces and fixed with $4 \%$ paraformaldehyde in $0.1 \mathrm{~m}$ phosphate buffer (PB, pH 7.2) for $24 \mathrm{~h}$. After an overnight wash in $0.1 \mathrm{M} \mathrm{PB}$ containing $20 \%$ sucrose, muscle was cut in the transverse plane at $16-20 \mu \mathrm{m}$ thickness on a cryostat and mounted on Superfrost Plus slides (Fisher). After drying and washing for $30 \mathrm{~min}$ in PBS, sections were incubated at room temperature with 500 nм Alexa Fluoro 555 -conjugated- $\alpha$-Btx for 30 min. After 3 washes with $0.1 \mathrm{M} \mathrm{PB} \mathrm{(each} 30$ $\mathrm{min})$, sections were mounted with coverslips using Fluoromount-G (Southern Biotechnology Associates). 
a.

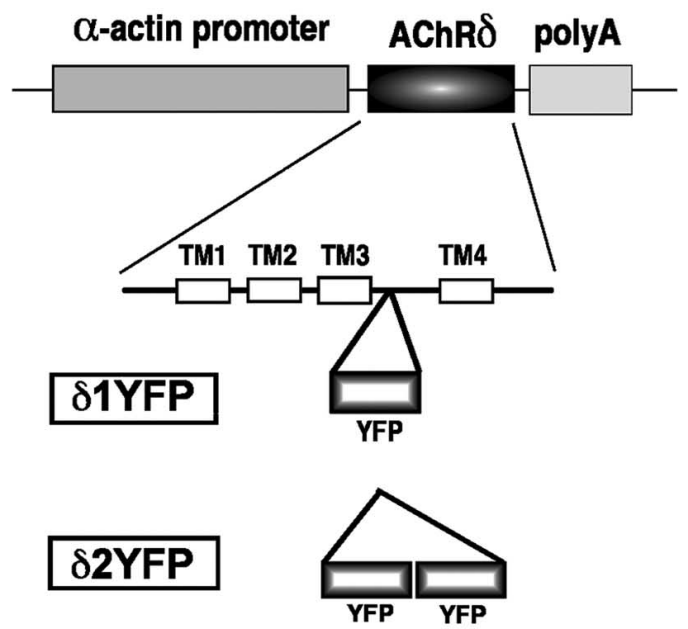

b.

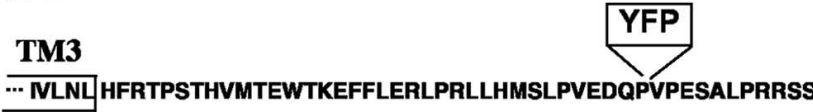

SLGYIAQAEEYYSVKSRSELMFEKQSERHGLAARPTPKATYTSSNDSEVSEQL

TM4

YNEMKPAVEGANYIVKHMHDKNDYNEEKDNWSGIARTVDRL CFYVVTPVM -..

C.

\section{Endogeneous $\delta$}

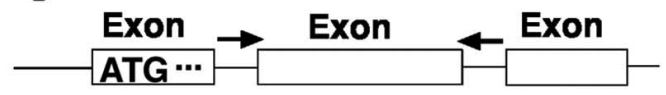

Wild type allele …СТСA $\cdots$ sop allele $\quad$...CCCCA ...

\section{Transgene}

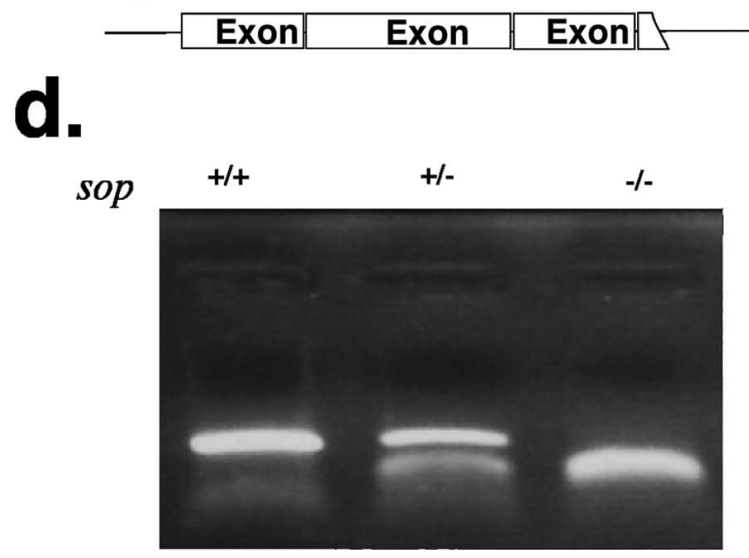

Figure 1. $\boldsymbol{a}$, Construct of $\delta$ YFP. A schematic of the DNA construct for the expression of the zebrafish AChR $\delta$-subunit clone in sop ${ }^{-1-}$ embryos. The $\delta$-subunit sequence has an insertion of YFP in the intracellular loop between transmembrane regions 3 and 4. $\delta 1 Y F P$, which has one YFP, and $\delta 2$ YFP, which has 2 YFPs, are shown. The fusion gene is flanked by the $\alpha$-actin promoter and the polyA addition signal. The expression of the fusion gene is driven by the muscle-specific $\alpha$-actin promoter. $\boldsymbol{b}$, Amino acid sequence of the long intracellular loop of the zebrafish $\delta$-subunit. 0 ne or two YFPs were inserted at the indicated site. TM3 and TM4 indicate transmembrane regions 3 and 4, respectively. c, PCR for genotyping sop ${ }^{-1-}$ embryos. Primers were designed to the introns of the $\delta$-subunit gene flanking the exon that contains the critical

\section{Results}

Introduction of $\delta 1 \mathrm{YFP}$ or $\delta 2 \mathrm{YFP}$ into sop ${ }^{-/-}$fish

A DNA construct was made to express the zebrafish $\delta$-subunit in $s o p^{-1-}$ background. The Megalinker sequence (Thermes et al., 2002), when used in conjunction with Meganuclease, increases the efficiency of genome integration. The $\delta$-subunit sequence is flanked by the $\alpha$-actin promoter (Higashijima et al., 1997) and a polyadenylation signal sequence. The $\delta$-subunit sequence contained either a single or double YFP sequence, such that the YFPs are inserted into the intracellular loop of the $\delta$-subunit (Fig. $1 a, b)$. The intracellular loop between transmembrane regions 3 and 4 was chosen as the insertion site based on previous studies of other nicotinic receptors (Nashmi et al., 2003).

81YFP or $\delta 2$ YFP was injected into zebrafish eggs obtained from a $s o p^{+/-}$male and $s o p^{+/-}$female cross. After $3 \mathrm{~d}$, YFP signals were observed in muscle cells of injected embryos in a stochastic pattern (Fig. $2 d$ ), i.e., some muscles express the introduced gene, whereas other muscle cells do not. This stochastic pattern arises from the random genome integration of injected DNA. A quarter of the embryos obtained from the $\operatorname{sop}^{+1-} \times$ sop ${ }^{+1-}$ cross were $\operatorname{sop}^{-1-}$, indicating Mendelian inheritance. Without the introduction of the $\delta$-subunit construct, the homozygous embryos are immotile when released from the chorion (Fig. 2c) (Ono et al., 2001). However, injected embryos that are genetically $s o p^{-1-}$ display muscle contraction as evidenced by a partial escape response following tactile stimuli. In this response, embryos display an initial bending of the trunk; however, the movement is not sustained, and the embryo cannot travel a long distance (Fig. 2a,b). The attenuated escape movement is likely caused by the stochastic nature of the $\delta 2$ YFP expression. To confirm the genotype of the embryos, genomic DNA was extracted from the embryos and PCR was performed (Fig. 1c). Primers were designed to regions within the introns of the $\delta$-subunit gene, thus enabling the amplification of genomic sequence without contamination from the injected $\delta$ YFP sequence. Amplicons were subsequently treated with a restriction enzyme, BccI. Amplicons generated from a wild-type allele remained undigested with BccI and exhibited a 397 bp band when run on an agarose gel. Conversely, amplicons derived from the mutant allele, sop $\mathrm{tj}^{19 \mathrm{~d}}$, were cut with $\mathrm{BccI}$ and gave rise to two shorter bands (181bps and 214bps) (Fig. 1d). Embryos with compromised motility were confirmed to be $s o p^{-1-}$.

AChRs containing $\delta 1$ YFP or $\delta 2$ YFP make normal clusters under nerve endings

Because $\delta 1 / 2$ YFP is fluorescent, we can visualize the localization of $\delta$-subunits in live embryos. In $s o p^{-1-}$ embryos injected with the construct, YFP fluorescence was detected in muscle cells in a stochastic pattern (Fig. $2 d$ ). Among cells that express the exogeneous $\delta$ YFP gene, the expression level of $\delta$ YFP, estimated by the YFP fluorescence intensity, was variable. Some cells expressed a large amount of protein and the YFP signal filled the cytoplasm (Fig. 3). Other cells exhibited a spatially restricted distribution,

$\leftarrow$

T to $C$ mutation of sop. The PCR product was generated only from the genomic sequence and not from the introduced transgene ( $\delta 1 / 2 Y F P)$. The PCR product was digested with Bccl to distinguish between amplicons from the wild-type allele and the sop allele. $d$, Electrophoresis of PCR products after Bccl digestion. When PCR products are treated with Bccl restriction enzyme and run on an agarose gel, sop ${ }^{+/+}$(wild type), sop ${ }^{+/-}$(heterozygous) and sop ${ }^{-1-}$ (homozygous) display distinct patterns. The upper band corresponds to 397 bps which is the full amplicon not digested with Bccl. The lower bands correspond to 181 bps and 214 bps, which are products of the $B c c$ digestion. 
resembling the synaptic distribution pattern of AChRs in wild-type zebrafish embryos (Fig. 3). These patterns likely represent the distribution of pentamers composed of $2 \alpha s, \beta, \gamma / \varepsilon$ and $\delta$ YFP subunits. To confirm this, we counter-stained these embryos with $\alpha$-Btx conjugated with Alexa555. $\alpha$-Btx binds to the $\alpha$-subunit at two sites, between the $\alpha$ - and $\gamma / \varepsilon$-subunits and the $\alpha$ - and $\delta$-subunits (Blount and Merlie, 1988). The clusters of YFP signals overlap with $\alpha$-Btx signals (Fig. 3). Furthermore, these clusters of AChRs are apposed to nerve endings, as indicated by costaining with SV2 antibody. SV2 is a protein involved in exocytosis at presynaptic terminals (Buckley and Kelly, 1985). Clusters of YFP and $\alpha$-Btx overlap with SV2 signals (Fig. 3). These results show that AChRs containing $\delta 1 / 2$ YFP form clusters at nerve endings in a similar manner to endogenous subunits in wild-type fish.

Establishment of sop ${ }^{-/-}$fish expressing 82YFP in all muscle cells

When embryos injected with the $\delta 2$ YFP construct mature, some of these fish (F0) harbor the transgene in germline cells. Embryos generated from such an adult (F1) harbor the transgene in all somatic cells, resulting in the expression of $\delta 2 \mathrm{YFP}$ in all muscle cells. Transgene expression is limited to muscle cells by the $\alpha$-actin promoter used in the DNA construct. Sop ${ }^{-1-}$ embryos with stable expression of $\delta 2$ YFP exhibit homogeneous expression of YFP in all muscle cells (Fig. 4a). Protein was extracted from these fish and Western blot analysis was performed with an antibody against GFP (also specific for YFP). A sin-

gle band was observed between $100 \mathrm{kDa}$ and $120 \mathrm{kDa}$, which corresponds to the molecular mass of $\delta 2$ YFP $(\sim 116 \mathrm{kDa})$. This band was not observed in protein extracted from wild-type fish (Fig. $4 b$ ). Embryos with stable expression of $\delta 2$ YFP also exhibit a much stronger response to tactile stimuli (Fig. $4 d$ ) than $s o p^{-1-}$ embryos with a stochastic expression of $\delta 1 / 2$ YFP (Fig. 2b). Sop ${ }^{-1-}$ stably expressing $\delta 2$ YFP display swimming behaviors close to those of wild-type embryos at $3 \mathrm{dpf}$. To compare movements of wild-type embryos and $\delta 2 \mathrm{YFP} /$ sop $^{-1-}$ embryos in more detail, their escape in response to a tail stimulus was recorded using a high-speed camera. Movements are represented as plots of rostral midlines (Fig. $4 d$ ). Traces of the midline movements did not show obvious differences. The head angle measured from these traces was plotted against time to compare parameters of the initial turn (Fig. $4 d$ ). In $\delta 2 \mathrm{YFP} / \mathrm{sop}^{-1-}$, the obtained parameters were: maximum angle $117 \pm 6$ degrees, peak angular velocity $24 \pm 2$ degrees $/ \mathrm{ms}$, duration $8.5 \pm 0.2 \mathrm{~ms}$ (mean $\pm \mathrm{SEM}, n=$ 12 trials from 3 embryos). The values for siblings without the transgene were: maximum angle $133 \pm 3$ degrees, peak angular velocity $25 \pm 1$ degrees $/ \mathrm{ms}$, duration $9.1 \pm 0.2 \mathrm{~ms}$ (mean \pm SEM, $n=14$ trials from 5 embryos). The maximum angle was slightly larger in control siblings and significantly different from $\delta 2 \mathrm{YFP} /$ sop ${ }^{-/-}$siblings (Student's $t$ test; $p<0.05$ ). However, the peak b. $\delta 2$ YFP in $s o p^{-/-}$
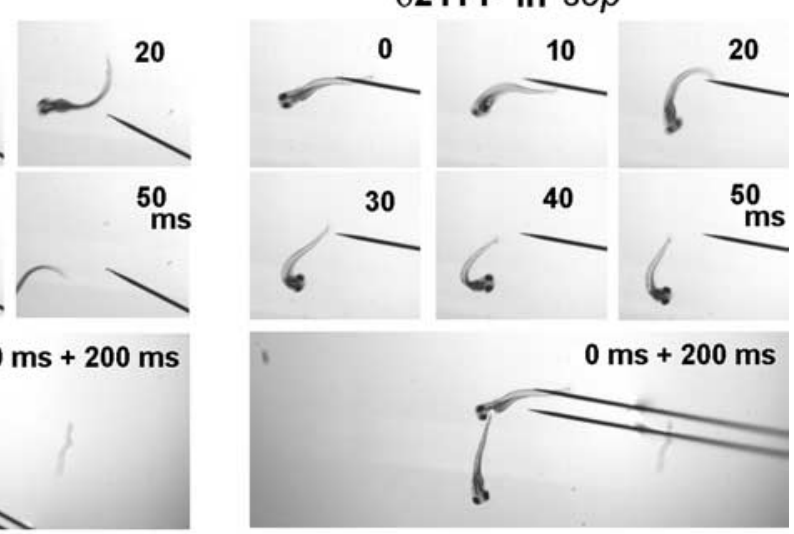

$0 \mathrm{~ms}+200 \mathrm{~ms}$

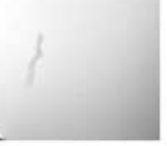

d.
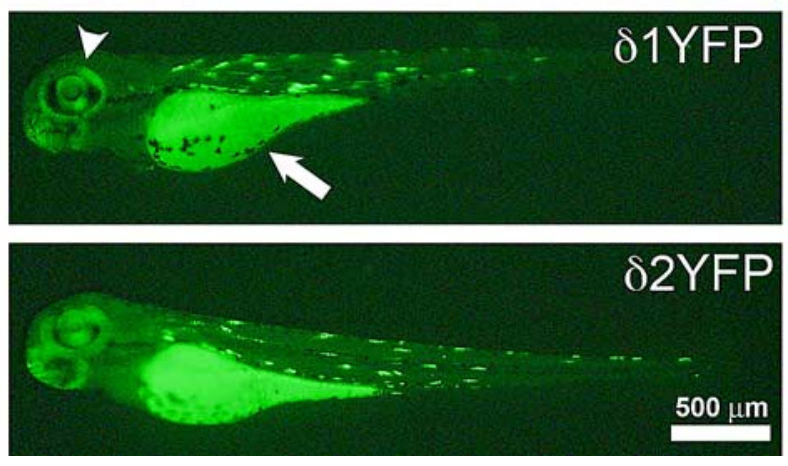

$500 \mu \mathrm{m}$

Figure 2. a, Movement of wild-type embryo. Sequential images showing movement of a $3 \mathrm{dpf}$ wild-type embryo in response to tactile stimulation. Each panel represents images taken at $0,10,20,30,40$, or 50 ms after the initiation of movements. $A$ embryo displayed in the same manner as $\boldsymbol{a} . \boldsymbol{c}_{\text {, Sop }}{ }^{-1-}$ embryo exhibits no voluntary movement at 0,50, or $200 \mathrm{~ms}$. $\boldsymbol{d}$, (he 1 cell stage. Fluorescence in the eye (arrowhead) and yolk sack (arrow) is endogenous. YFP signal resulting from the expression of $\delta 1 / 2$ YFP is detected in muscle cells in the trunk. Expression occurs in a stochastic manner, and the fluorescence is mosaic.

angular velocity and the duration were not different $(p>0.05)$. Despite this small difference at $3 \mathrm{dpf}, \delta 2 \mathrm{YFP} / s o p^{-1-}$ embryos follow a normal course of development. $\delta 2 \mathrm{YFP} / \operatorname{sop}^{-1-}$ embryos develop swim bladders at 4-5 dpf, a phenotype never observed in sop ${ }^{-1-}$ embryos lacking the transgene (Fig. $4 c$ ).

The expression pattern of $\delta 2$ YFP in stable lines was uniform among individual muscle cells (Fig. 4a), unlike embryos with a stochastic expression (Figs. 2, 3). Although some muscle cells express a low level of YFP in cytoplasm early in development, the majority of YFP signals were localized to the plasma membrane and form clusters at synapses (Fig. $5 a$ ). The pattern of YFP clusters resembles that of AChR distribution in wild-type embryos when visualized by $\alpha$-Btx staining.

We compared synaptic currents resulting from AChRs containing 82 YFP to those of wild-type fish. Miniature end plate currents (mEPCs) were recorded from muscle cells of embryos by voltage clamping at $-90 \mathrm{mV}$. Skeletal muscle cells in zebrafish embryos are categorized into two groups: fast and slow twitch fibers (Pette and Staron, 1990; Devoto et al., 1996). Fast and slow fibers in fish correspond to those in mammals although there are some important differences. First, slow fibers in zebrafish do not fire action potentials, whereas mammalian slow fibers do (McArdle et al., 1980; Buckingham and Ali, 2004). Second, mam- 
$\delta 1 Y F P$
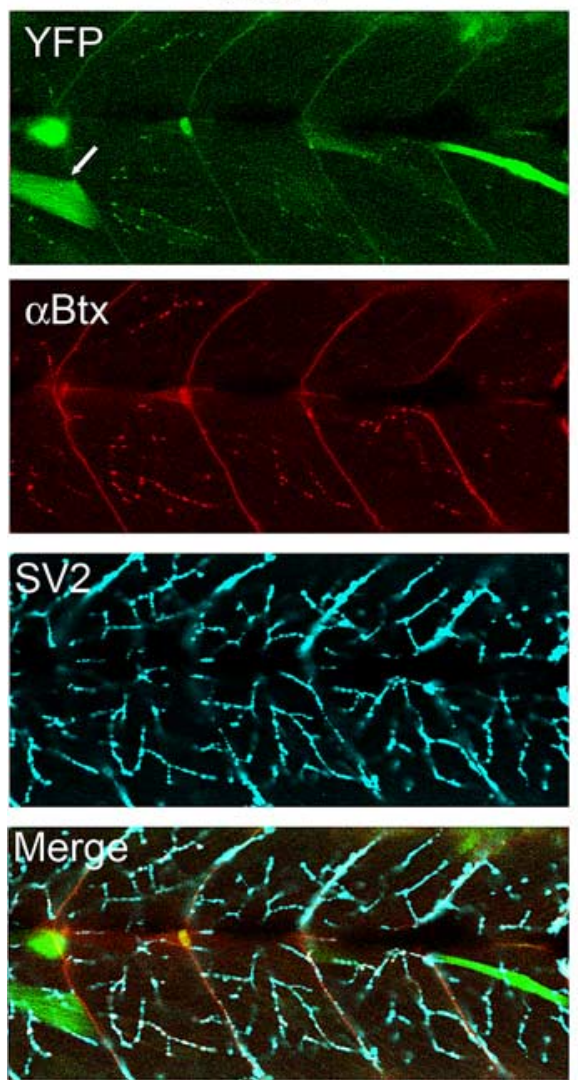

$\delta 2 Y F P$
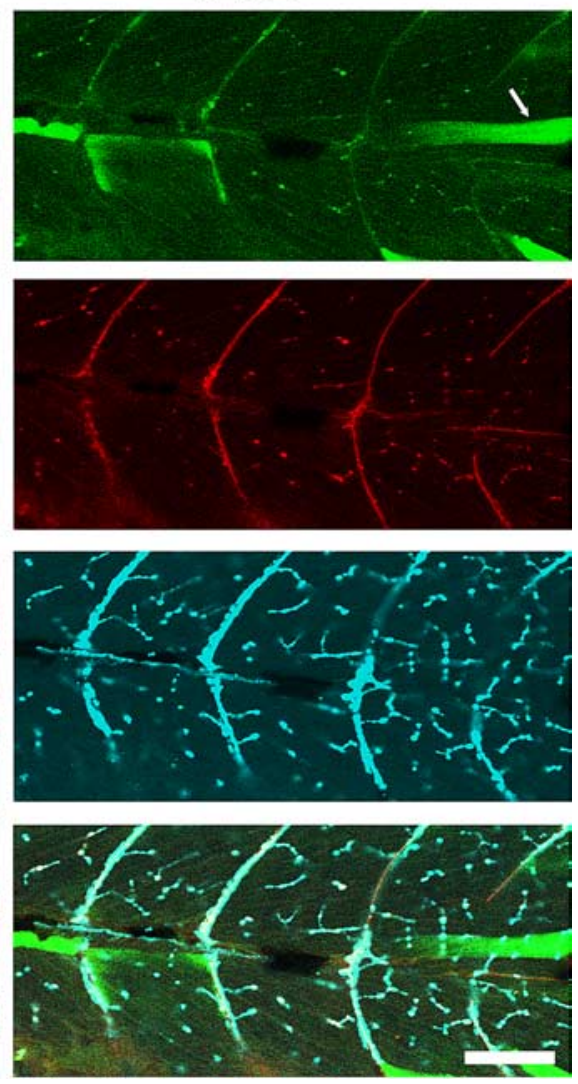

Figure 3. Clustering of AChRs comprised of $2 \alpha, \beta, \gamma / \varepsilon$ and $\delta 1 Y F P$ or $\delta 2 Y F P$ subunits. Confocal images of sop ${ }^{-1-}$ embryos expressing $\delta 1$ YFP (left) or $\delta 2$ YFP (right) were taken at $3 \mathrm{dpf}$. Top panels show the YFP signal. Whereas some muscle cells with high expression of $\delta 1 / 2$ YFP have cytoplasm filled with YFP signals (arrow), cells expressing lower level of $\delta$ YFP exhibit clusters. These clusters of YFP colocalize with $\alpha$-BTX signals (second row). In the third row, nerve terminals are visualized by anti-SV2 antibody. In muscle cells that express $\delta 1 / 2$ YFP, YFP clusters, $\alpha$-Btx and SV2 staining overlap and display white color in the merged picture (bottom). Scale bar, $50 \mu \mathrm{m}$.

malian muscle is a mixture of these two types, whereas in teleosts they are spatially segregated and can be identified visually under the microscope (Buss and Drapeau, 2002). The mEPCs recorded from each type are different, thus mEPCs from each cell type were compared separately. In wild-type embryos, the current amplitude was $879 \pm 67 \mathrm{pA}($ mean \pm SEM $)$ for slow muscle and $464 \pm$ $22 \mathrm{pA}$ for fast muscle. The rise time was $0.40 \pm 0.04 \mathrm{~ms}$ for slow muscle and $0.25 \pm 0.02 \mathrm{~ms}$ for fast muscle. The decay time constant of a single exponential fitting was $1.70 \pm 0.08 \mathrm{~ms}$ for slow muscle and $0.63 \pm 0.02 \mathrm{~ms}$ for fast muscle $(n=87$ from 4 slow muscle cells, $n=89$ from 3 fast muscle cells). When mEPCs were recorded from fast and slow muscles in $\delta 2 \mathrm{YFP} / \mathrm{sop}^{-/-}$embryos, the current amplitude was $178 \pm 18 \mathrm{pA}$ for slow muscle and $95 \pm$ $11 \mathrm{pA}$ for fast muscle. The rise time was $0.57 \pm 0.03 \mathrm{~ms}$ for slow muscle and $0.31 \pm 0.01 \mathrm{~ms}$ for fast muscle. The decay time constant was $1.99 \pm 0.07 \mathrm{~ms}$ for slow muscle and $0.71 \pm 0.02 \mathrm{~ms}$ for fast muscle ( $n=101$ from 3 slow muscle cells, $n=159$ from 3 fast muscle cells). The rise time and decay time constant were slower in $\delta 2 \mathrm{YFP} / \mathrm{sop}^{-1-}$ embryos compared with wild-type embryos, both in fast muscle and slow muscle $(p<0.05)$ (Fig. 5e). The difference of the current amplitude was more pronounced: the current amplitude in $\delta 2 \mathrm{YFP} / \mathrm{sop}^{-1-}$ was smaller than that of wild-type fish, both in fast and slow muscle $(p<0.05)$ (Fig. $5 e$ ).

When extracellular stimulation was applied to the spinal cord, end plate current (EPC) was recorded from voltage clamped muscle cells. The time course of the synaptic currents was com- parable with those from wild-type embryos (Fig. $5 d$ ). This indicates that the presynaptic machinery of vesicle release is intact in $\delta 2 \mathrm{YFP} / \mathrm{sop}^{-1-}$ embryos. Normal vesicle release was expected because presynaptic vesicle recycling is intact even in the complete absence of $\delta$-subunits. This is evidenced by a study of $s o p^{-1-}$ embryos using FM1-43 (Li et al., 2003).

\section{S2YFP/sop ${ }^{-/-}$embryos survive beyond sexual maturation}

$\delta 2 \mathrm{YFP} / \mathrm{sop}^{-/-}$embryos grow-up normally to full sexual maturation, which is $\sim 3$ months after hatching. Because these fish cannot be distinguished from wildtype or $s o p^{+/-}$siblings, they need to be identified as $\delta 2 \mathrm{YFP} / \mathrm{sop}^{-/-}$by genotyping with tail-clippings. A sexually mature, 12month-old fish, identified as $\delta 2 \mathrm{YFP} /$ $s o p^{-/-}$, is shown in Figure 6. Its genotype was confirmed by two methods: using BccI and direct sequencing (Fig. 6b). The fish was anatomically normal (Fig. 6a), and displayed swimming behaviors close to age-matched wild-type fish (Fig. $6 c$ ). This fish was able to mate normally and produced offspring. Normal development of $\delta 2 \mathrm{YFP} / \mathrm{sop}^{-/-}$embryos occurred in multiple lines arising from independent founder fish (F0), indicating the locus of genome integration is not critical. The transgene continued to be expressed and the YFP signal was maintained in the $\mathrm{NMJ}$ of the $\delta 2 \mathrm{YFP} / \mathrm{sop}{ }^{-1-}$ fish (Fig. $6 d$ ). In the cross-sectioned muscle, YFP signals were observed in the periphery of individual muscle fibers, and these YFP signals overlapped with $\alpha$-Btx staining. Thus, the introduced $\delta 2$ YFP continues to express and function in adults supporting the normal development and behavior of these transgenic fish.

\section{Discussion}

In the current study, we established transgenic zebrafish whose synaptic transmissions in the NMJ depend exclusively on an exogeneously introduced AChR subunit. A modified $\delta$-subunit was expressed in the background of a zebrafish null-mutant line, sofa potato $\left(s o p^{-1-}\right)$. The introduced $\delta$-subunit has one or two YFP molecules fused to the intracellular loop. We examined the development of the NMJ and synaptic current in rescued embryos.

Green fluorescent proteins (GFP), originally isolated from jellyfish (Shimomura et al., 1962), are now widely used in the biomedical field. They are particularly useful when fused to other proteins, rendering them visible in vivo (Tsien, 1998). We previously fused GFP to rapsyn to visualize rapsyn molecules at the NMJ in vivo. The disruption of rapsyn function was minimal, as evidenced by the ability of the fusion protein to rescue the phenotype of rapsyn-null mutants (Ono et al., 2002). Channels and receptors also have been fused to fluorescent molecules, but their functions are more easily disrupted (Fucile et al., 2002). Fluorescent molecules have been added to the intracellular loop of some genes in the AChR family, including $\alpha 4, \alpha 6, \beta 2$ and $\beta 3$ (Nashmi et al., 2003; Drenan et al., 2008). Integration at this site resulted in 
the least disturbance of the AChR channel properties (Nashmi et al., 2003). Interestingly, this intracellular loop between the transmembrane regions 3 and 4 of the subunit plays a critical role for the trafficking of some AChR molecules. For example, swapping the intracellular loop of the $\alpha 7$ subunit with that of the $\alpha 3$-subunit changes its trafficking. $\alpha 7$, which is localized perisynaptically in ciliary ganglion neurons, is now directed to the synapse (Williams et al., 1998). In terms of synapse formation, we did not see any effect of the YFP addition to the $\delta$-subunit. $\delta 1$ YFP or $\delta 2$ YFP form pentamers with the other receptor subunits, as evidenced by $\alpha$-Btx staining. They also make synaptic clusters comparable with those in wild-type fish, with the clusters found apposed to the motor nerve terminals (Fig. 3). Thus, the addition of one or two YFPs to the long intracellular loop did not produce any effect on the trafficking of $\delta$-subunits or assembled pentamers.

Fluorescence intensity of $\delta 1 Y F P$ and $\delta 2$ YFP was not obviously different when observed under the same optical condition (Fig. $2 d$ ). In theory, 82 YFP is expected to emit twice the amount of photons per single AChR pentamer compared with $\delta 1 Y F P$. However, in stochastic expression, the level of protein expression is variable from cell to cell, and the difference in signal strength per molecule may be obscured by the larger variability of the expression level. Alternatively, one YFP molecule in the 82YFP-subunit may not be fluorescent.

Luna and Brehm (2006) performed a careful analysis of synaptic currents in slow and fast fibers, using intracellular recording as well as extracellular field current measurements. The rise time and decay time were slower in slow fibers. A similar difference was also observed in wild-type larvae in this study, although larvae used in this study were younger compared with those used by Luna and Brehm (2006). Synaptic currents from $\delta 2 \mathrm{YFP} / \mathrm{sop}^{-1-}$ stable lines showed small differences from wild-type embryos in rise time and decay time constant. The difference between wild-type and $\delta 2 \mathrm{YFP} /$ sop ${ }^{-1-}$ was more pronounced in the current amplitude. The maximum currents generated by $\delta 2 \mathrm{YFP} / \mathrm{sop}^{-1-}$ were close to those of wild type, but the amplitude distribution was different, leading to a different average (Fig. 5). It was reported that the fusion of fluorescent molecules to some nicotinic receptors changes the receptor kinetics (Fucile et al., 2002; Palma et al., 2002). The slower rise time and decay time may reflect the change of rate constants between channel states. The smaller amplitude may arise from a smaller conductance of individual channels. Alternatively it may result from a reduction in

\section{Wild type} is different from trial to trial.
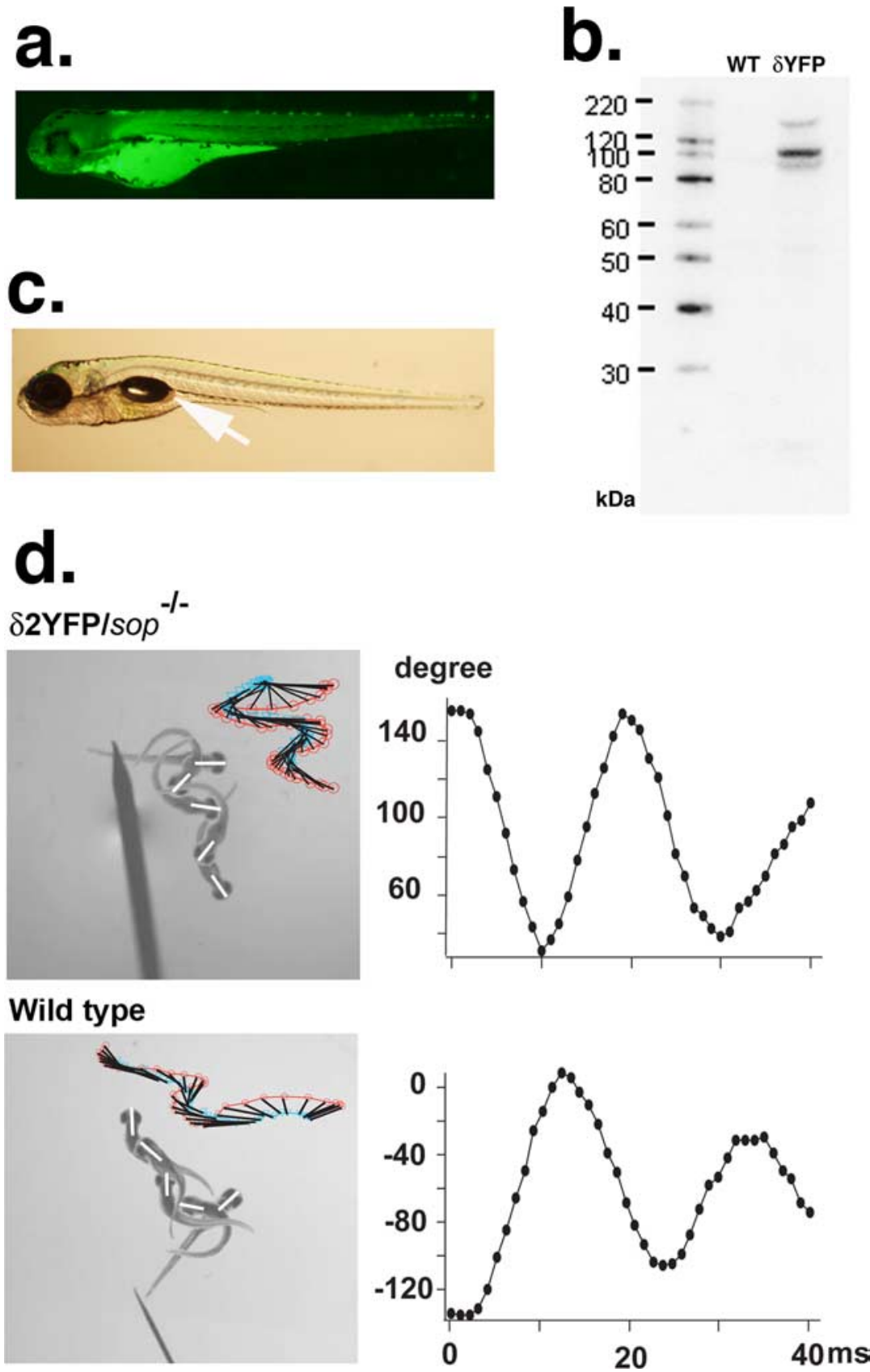

Figure 4. $\boldsymbol{a}, \mathrm{YFP}$ fluorescence of a $3 \mathrm{dpf}$ sop ${ }^{-1-}$ embryo stably expressing $\delta 2 \mathrm{YFP}\left(\delta 2 \mathrm{YFP} / \mathrm{sop}^{-1-}\right)$ in all muscle cells. $\boldsymbol{b}$, Western blot of $\delta 2 \mathrm{YFP}$ protein using antibody against YFP. A band is observed $\sim 110 \mathrm{kDa}$ in $\delta 2 \mathrm{YFP} / \mathrm{sop}^{-1-}$ embryos. A corre-

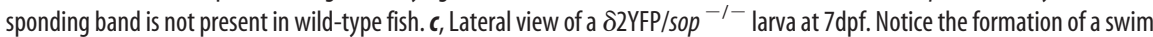
bladder (arrow). Sop ${ }^{-1-}$ embryos without the transgene never form swim bladders. $\boldsymbol{d}$, Movement of embryos at $3 \mathrm{dpf}$ in response to a tail stimulus. A $\delta 2 \mathrm{YFP} /$ sop $^{-1-}$ embryo is shown in the top and a wild-type embryo (sop ${ }^{+1+}$ without the transgene) is shown in the bottom. Superimposed images of embryos at 0,10,20,30, and 40 ms are shown to the left. Rostral midlines at each time point are shown as white lines. Insets represent swimming as a series of rostral midlines. The beginning of the line corresponding to the head is shown as red circles and the end of the line is shown as blue triangles. Head angles plotted against time for each trace are shown on the right. Note the initial angle is determined by the positioning of the embryo before touch and

the receptor number at the synapse because of the reduced expression level of $\delta 2$ YFP compared with the endogenous $\delta$. Overall, however, the effect of YFP fusion on the synaptic current was unexpectedly mild, even with the addition of two YFP molecules. Further analysis at the single channel level from $\delta 2 \mathrm{YFP} /$ sop $^{-1-}$ fish will determine the nature of the altered channel kinetics.

Despite these synaptic current changes, embryos did not dis- 
a.

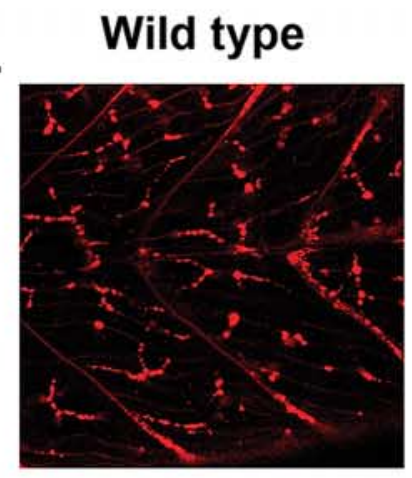

b.

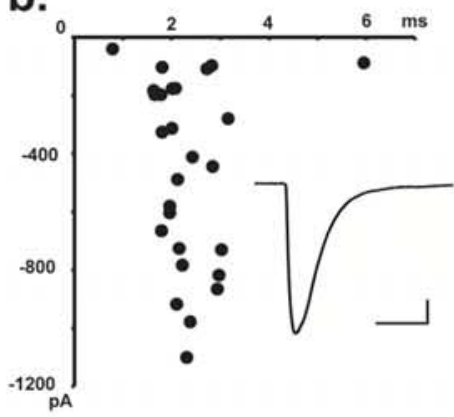

C.

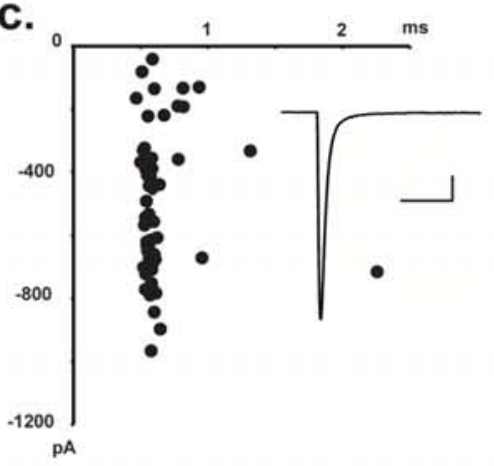

d.

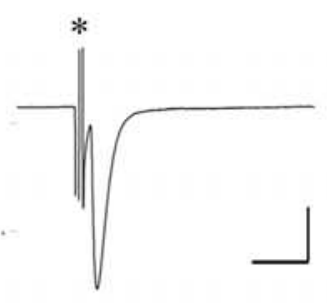

$\delta 2$ YFP / sop s- $^{-1-}$
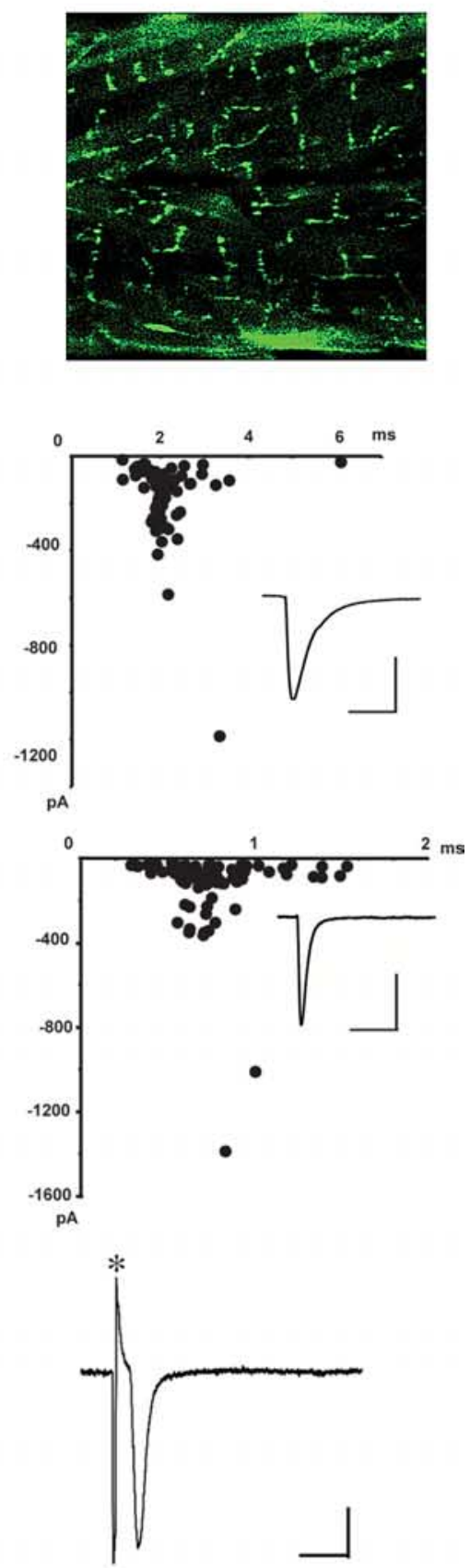

e.

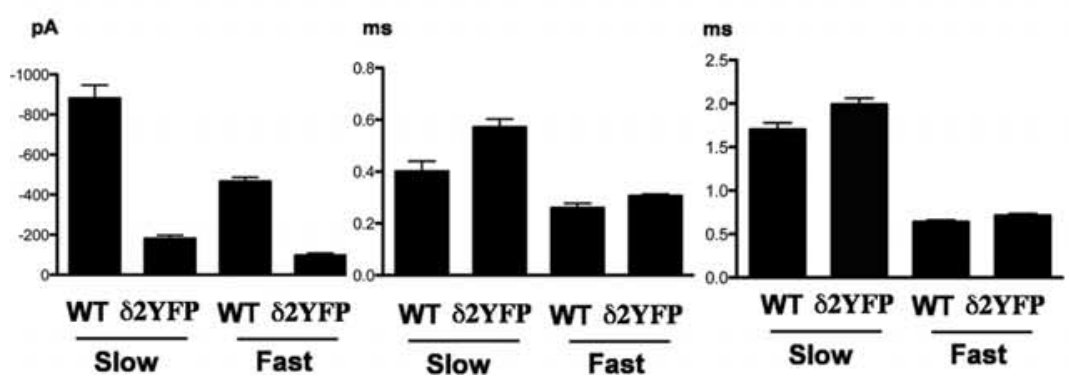

Figure 5. $\quad \boldsymbol{a}$, Confocal images showing the distribution of AChR clusters in wild-type embryos visualized by $\alpha$-Btx (left), and the distribution of AChR clusters visualized by YFP in $\delta 2 \mathrm{YFP} / \mathrm{sop}^{-1-}$ embryo, stably expressing $\delta 2 \mathrm{YFP}$ (right). Both images were obtained at $3 \mathrm{dpf}$. $\boldsymbol{b}$, mEPCs recorded from a single slow-twitch muscle cell of a wild-type embryo (left) and a $\delta 2 \mathrm{YFP} / \mathrm{sop}^{-1-}$ embryo (right) at $3 \mathrm{dpf}$. Amplitude and decay time constant of individual mEPCs are plotted. Averaged traces are shown as insets. play noticeable abnormality in behavior throughout development. Introduction of $\delta 2$ YFP into sop ${ }^{-/-}$embryos rescued the swimming behavior in the stable line. The swimming pattern is close to normal, and analysis at $1000 \mathrm{fps}$ revealed only a small difference in the escape response at $3 \mathrm{dpf}$. A hallmark of normal development in $\delta 2 \mathrm{YFP} / \mathrm{sop}^{-1-}$ embryos is the development of a swim bladder (Fig. 4c). Many zebrafish locomotion mutants fail to develop swim bladders. For example, shocked mutants fail to develop swim bladders, despite its ostensible recovery of swimming behavior after initial swimming defects (Luna et al., 2004; Cui et al., 2005). This shows that the swimming behavior of $\delta 2 \mathrm{YFP} / \mathrm{sop}^{-1-}$ embryos is better than that of the "recovering" locomotion mutants.

Indeed, $\delta 2 \mathrm{YFP} / \mathrm{sop}^{-1-}$ embryos survive to adulthood. They display no obvious anatomical abnormality (Fig. 6a), whereas human embryos lacking $\delta$-subunits exhibit an array of anatomical defects including nuchal hygroma, pterygia, contractures, and scoliosis (Michalk et al., 2008). In terms of movement, $\delta 2 \mathrm{YFP} /$ sop ${ }^{-1-}$ adult fish perform normal locomotion including a C-shaped bending of the body, which is important for the change of swimming directions in teleosts (Fig. 6c). As a result, they can perform activities for survival. Eating flake food on the water surface requires a highly coordinated movement of different parts of the body, which $\delta 2 \mathrm{YFP} / \mathrm{sop}{ }^{-1-}$ can perform without difficulty (Fig. 6c). Normal zebrafish reach sexual maturity $\sim 3$ months after hatching. $\delta 2 \mathrm{YFP} / \mathrm{sop}^{-1-}$ can perform a normal mating behavior and generate viable offspring. Thus, in every aspect we observed, $\delta 2 \mathrm{YFP} / \mathrm{sop}^{-1-}$ displays movements and behaviors close to that of wildtype fish, although its synaptic transmission is dependent on the 82 YFP throughout development.

When expressed in Xenopus oocytes, mammalian AChR subunits function in the absence of $\delta$-subunit, although with a

\footnotetext{
Calibration: $50 \mathrm{pA}, 6 \mathrm{~ms} . \mathrm{c}, \mathrm{mEPC}$ recorded from a single fasttwitch muscle cell of a wild-type embryo (left) or a $\delta 2 Y F P /$ sop $^{-1-}$ embryo (right). Amplitude and decay time constant of individual $m E P C s$ are plotted. Averaged traces are shown as insets. Calibration: $50 \mathrm{pA}, 6 \mathrm{~ms}$. $\boldsymbol{d}$, EPC recordings in response to spinal cord stimulation. Stimulation artifacts are marked with asterisks. Calibration: $500 \mathrm{pA}$ (wild type), $200 \mathrm{pA}\left(\delta 2 \mathrm{YFP} / \mathrm{sop}^{-/-}\right.$), $5 \mathrm{~ms}$. $\boldsymbol{e}$, Accumulated data of fast-twitching and slow-twitching muscle cells from wild-type and $\delta 2 \mathrm{YFP} / \mathrm{sop}^{-1-}$ embryos. Current amplitude (left), rise time (middle) and decay time constant (right) of mEPCs are shown. Bar graphs represent the mean, and error bars are SEM.
}

$\leftarrow$ 
reduced conductance (Kullberg et al., 1990; Liu and Brehm, 1993). Interestingly, $\delta$-less receptors form with $\gamma$-subunit (embryonic-type), but not with $\varepsilon$-subunit (adult-type). Therefore, human embryos lacking the $\delta$-subunit may exhibit low conductance AChR current at NMJ before the $\gamma$-to- $\varepsilon$ switch is complete. Weakness of the synaptic current or the failure of transition to adult-type current may be the cause of FADS symptoms and the premature death in human. Frogs are different from mammals in that their $\delta$-subunit is necessary for the functional AChR formation (Paradiso and Brehm, 1998). It is clear that zebrafish also need the $\delta$-subunit, because in the absence of a functional $\delta$-subunit, the NMJ in $s o p^{-1-}$ does not display a synaptic current (Ono et al., $2001)$. The survival of the $\delta 2 \mathrm{YFP} / \mathrm{sop}^{-1-}$ fish shows that even such severe defects of NMJ can be rescued.

The normal development of $\delta 2 \mathrm{YFP} /$ $s o p^{-1-}$ was unexpected for two reasons. First, the synaptic current is changed, most notably in the current amplitude. The NMJ is believed to have a safety margin to ensure the faithful contraction of muscle in response to nerve stimuli. However, in some forms of CMD, the compromised current amplitude leads to symptoms in the skeletal muscle. The normal development and behavior of the $\delta 2 \mathrm{YFP} / \mathrm{sop} \mathrm{p}^{-1-}$ fish indicates that the altered synaptic current causes no deleterious effect on the animal throughout development. Second, the expression of $\delta 2 \mathrm{YFP}$ is regulated not by the native promoter of the $\delta$-subunit gene but by the $\alpha$-actin promoter. The expression of $\delta 2 \mathrm{YFP}$ will be different from that of the endogenous $\delta$-subunit in terms of timing and quantity. Many genes that play roles in development are regulated spatially and temporally by factors in cellautonomous or non-autonomous manner. AChR subunit transcripts become concentrated to synaptic sites with innervation (Burden, 1998). In mice, the $\delta$-subunit gene becomes selectively transcribed at synaptic nuclei, which is mediated by the $\mathrm{N}$ box sequence in the promoter (Schaeffer et al., 2001). The normal development of $\delta 2 \mathrm{YFP} / s o p^{-1-}$ indicates that the strict regulation of the $\delta$-subunit gene is not essential for animal development. It may be that only one of the subunits comprising the pentamer needs to be strictly regulated.

The larger than expected flexibility of the NMJ to enable normal development of animals may be useful for future studies. First, the addition of two YFP molecules to the intracellular loop of the AChR $\delta$-subunit leads to a minimal functional defect at NMJs in vivo. Similar strategies will enable optical studies that require attachment of a large-sized molecule to receptors, such as chameleon (Miyawaki et al., 1997), in a living fish. It may even

a. b.

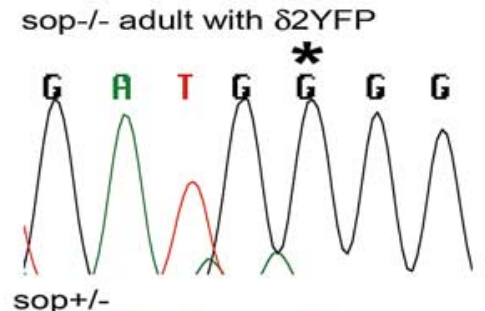

Wild type

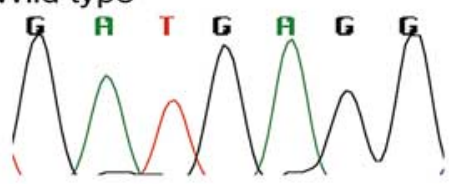

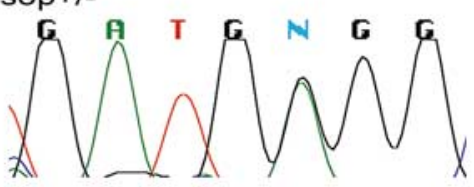

d.

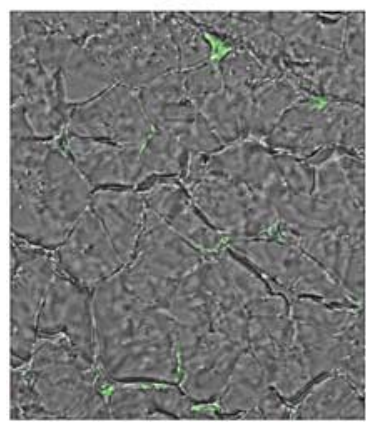

c.

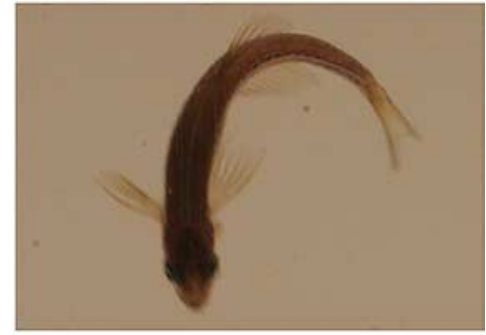

Figure 6. $\quad \boldsymbol{a}, \mathrm{A} 1$-year-old $\delta 2 \mathrm{YFP} / \mathrm{sop}^{-1-}$ fish. The fish is anatomically indistinguishable from wild-type fish. Scale bar, $1 \mathrm{~cm}$. $\boldsymbol{b}$, The $\delta 2 \mathrm{YFP} / \mathrm{sop}^{-1-}$ fish was genotyped with $\mathrm{Bccl}$ and direct sequencing. The chromatogram of sequencing results is shown, which is complementary to the coding strand. This fish shows $\mathrm{G}$ at the critical site (marked with an asterisk; top), whereas a heterozygous fish shows a mixture of $A$ and $G$ (middle), and a wild-type fish shows $A$ (bottom). c, Behaviors of a $\delta 2 Y F P /$ sop ${ }^{-1-}$ fish. In the top, it is making a C-bend. In the bottom, it is eating a flake of food on the water surface. $d$, YFP fluorescence detected from a transverse section of muscle in $\delta 2 \mathrm{YFP} / \mathrm{sop}^{-1-}$ fish. The left panel is a merged image of the YFP signal and transmitted light. The middle panel is a merged image of the $\alpha$-Btx signal and transmitted light. The right panel is the merged image of all signals.
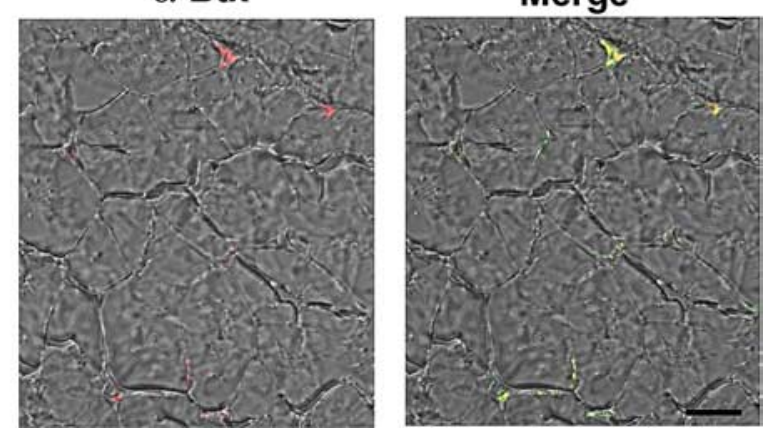

allow the visualization of receptor molecules in different states, using biophysical techniques that are sensitive to the relative orientation of two fluorescent molecules (Vogel et al., 2006). Second, it may provide a useful model system to study genetic diseases affecting NMJ, including FADS and CMD. Normal development of $\delta 2 \mathrm{YFP} /$ sop $^{-1-}$ fish suggest that the synapse is flexible enough for a replacement gene with altered transcriptional regulation and channel kinetics to support the survival of the animal. Further studies may delineate requirements for gene therapies of diseases affecting the NMJ. 


\section{References}

Blount P, Merlie JP (1988) Native folding of an acetylcholine receptor alpha subunit expressed in the absence of other receptor subunits. J Biol Chem 263:1072-1080.

Buckingham SD, Ali DW (2004) Sodium and potassium currents of larval zebrafish muscle fibres. J Exp Biol 207:841-852.

Buckley K, Kelly RB (1985) Identification of a transmembrane glycoprotein specific for secretory vesicles of neural and endocrine cells. J Cell Biol 100:1284-1294.

Burden SJ (1998) The formation of neuromuscular synapses. Genes Dev 12:133-148.

Buss RR, Drapeau P (2002) Activation of embryonic red and white muscle fibers during fictive swimming in the developing zebrafish. J Neurophysiol 87:1244-1251.

Cui WW, Low SE, Hirata H, Saint-Amant L, Geisler R, Hume RI, Kuwada JY (2005) The zebrafish shocked gene encodes a glycine transporter and is essential for the function of early neural circuits in the CNS. J Neurosci 25:6610-6620.

Devoto SH, Melançon E, Eisen JS, Westerfield M (1996) Identification of separate slow and fast muscle precursor cells in vivo, prior to somite formation. Development 122:3371-3380.

Drenan RM, Nashmi R, Imoukhuede P, Just H, McKinney S, Lester HA (2008) Subcellular trafficking, pentameric assembly, and subunit stoichiometry of neuronal nicotinic acetylcholine receptors containing fluorescently labeled alpha6 and beta3 subunits. Mol Pharmacol 73:27-41.

Engel AG, Sine SM (2005) Current understanding of congenital myasthenic syndromes. Curr Opin Pharmacol 5:308-321.

Fucile S, Palma E, Martinez-Torres A, Miledi R, Eusebi F (2002) The singlechannel properties of human acetylcholine alpha 7 receptors are altered by fusing alpha 7 to the green fluorescent protein. Proc Natl Acad Sci U S A 99:3956-3961.

Granato M, van Eeden FJ, Schach U, Trowe T, Brand M, Furutani-Seiki M, Haffter P, Hammerschmidt M, Heisenberg CP, Jiang YJ, Kane DA, Kelsh RN, Mullins MC, Odenthal J, Nüsslein-Volhard C (1996) Genes controlling and mediating locomotion behavior of the zebrafish embryo and larva. Development 123:399-413.

Higashijima S, Okamoto H, Ueno N, Hotta Y, Eguchi G (1997) Highfrequency generation of transgenic zebrafish which reliably express GFP in whole muscles or the whole body by using promoters of zebrafish origin. Dev Biol 192:289-299.

Kullberg R, Owens JL, Camacho P, Mandel G, Brehm P (1990) Multiple conductance classes of mouse nicotinic acetylcholine receptors expressed in Xenopus oocytes. Proc Natl Acad Sci U S A 87:2067-2071.

Li W, Ono F, Brehm P (2003) Optical measurements of presynaptic release in mutant zebrafish lacking postsynaptic receptors. J Neurosci 23:10467-10474.

Liu KS, Fetcho JR (1999) Laser ablations reveal functional relationships of segmental hindbrain neurons in zebrafish. Neuron 23:325-335.

Liu Y, Brehm P (1993) Expression of subunit-omitted mouse nicotinic acetylcholine receptors in Xenopus laevis oocytes. J Physiol 470:349-363.

Luna VM, Brehm P (2006) An electrically coupled network of skeletal muscle in zebrafish distributes synaptic current. J Gen Physiol 128:89-102.

Luna VM, Wang M, Ono F, Gleason MR, Dallman JE, Mandel G, Brehm P (2004) Persistent electrical coupling and locomotory dysfunction in the zebrafish mutant shocked. J Neurophysiol 92:2003-2009.

McArdle JJ, Michelson L, D’Alonzo AJ (1980) Action potentials in fast- and slow-twitch mammalian muscles during reinnervation and development. J Gen Physiol 75:655-672.

Michalk A, Stricker S, Becker J, Rupps R, Pantzar T, Miertus J, Botta G, Naretto VG, Janetzki C, Yaqoob N, Ott CE, Seelow D, Wieczorek D, Fiebig B, Wirth B, Hoopmann M, Walther M, Körber F, Blankenburg M, Mundlos S, Heller R, Hoffmann K (2008) Acetylcholine receptor pathway mutations explain various fetal akinesia deformation sequence disorders. Am J Hum Genet 82:464-476.

Mishina M, Takai T, Imoto K, Noda M, Takahashi T, Numa S, Methfessel C, Sakmann B (1986) Molecular distinction between fetal and adult forms of muscle acetylcholine receptor. Nature 321:406-411.

Miyawaki A, Llopis J, Heim R, McCaffery JM, Adams JA, Ikura M, Tsien RY (1997) Fluorescent indicators for $\mathrm{Ca} 2+$ based on green fluorescent proteins and calmodulin. Nature 388:882-887.

Nashmi R, Dickinson ME, McKinney S, Jareb M, Labarca C, Fraser SE, Lester HA (2003) Assembly of alpha4beta2 nicotinic acetylcholine receptors assessed with functional fluorescently labeled subunits: effects of localization, trafficking, and nicotine-induced upregulation in clonal mammalian cells and in cultured midbrain neurons. J Neurosci 23:11554-11567.

Ono F (2008) An emerging picture of synapse formation: a balance of two opposing pathways. Sci Signal 1:pe3.

Ono F, Higashijima S, Shcherbatko A, Fetcho JR, Brehm P (2001) Paralytic zebrafish lacking acetylcholine receptors fail to localize rapsyn clusters to the synapse. J Neurosci 21:5439-5448.

Ono F, Shcherbatko A, Higashijima S, Mandel G, Brehm P (2002) The zebrafish motility mutant twitch once reveals new roles for rapsyn in synaptic function. J Neurosci 22:6491-6498.

Ono F, Mandel G, Brehm P (2004) Acetylcholine receptors direct rapsyn clusters to the neuromuscular synapse in zebrafish. J Neurosci 24:5475-5481.

Palma E, Mileo AM, Martinez-Torres A, Eusebi F, Miledi R (2002) Some properties of human neuronal alpha 7 nicotinic acetylcholine receptors fused to the green fluorescent protein. Proc Natl Acad Sci U S A 99:3950-3955.

Paradiso K, Brehm P (1998) Long-term desensitization of nicotinic acetyl choline receptors is regulated via protein kinase A-mediated phosphorylation. J Neurosci 18:9227-9237.

Pette D, Staron RS (1990) Cellular and molecular diversities of mammalian skeletal muscle fibers. Rev Physiol Biochem Pharmacol 116:1-76.

Sanes JR, Lichtman JW (2001) Induction, assembly, maturation and maintenance of a postsynaptic apparatus. Nat Rev Neurosci 2:791-805.

Schaeffer L, de Kerchove d'Exaerde A, Changeux JP (2001) Targeting transcription to the neuromuscular synapse. Neuron 31:15-22.

Shimomura O, Johnson FH, Saiga Y (1962) Extraction, purification and properties of aequorin, a bioluminescent protein from the luminous hydromedusan, Aequorea. J Cell Comp Physiol 59:223-239.

Thermes V, Grabher C, Ristoratore F, Bourrat F, Choulika A, Wittbrodt J, Joly JS (2002) I-SceI meganuclease mediates highly efficient transgenesis in fish. Mech Dev 118:91-98.

Tsien RY (1998) The green fluorescent protein. Annu Rev Biochem 67:509-544.

Vogel SS, Thaler C, Koushik SV (2006) Fanciful FRET. Sci STKE 2006:re2.

Williams BM, Temburni MK, Levey MS, Bertrand S, Bertrand D, Jacob MH (1998) The long internal loop of the alpha 3 subunit targets nAChRs to subdomains within individual synapses on neurons in vivo. Nat Neurosci 1:557-562. 